\title{
A Simple and Noninvasive Zebrafish Egg Infection Model for Studying Pathogenic Yeasts
}

\author{
Yin-Zhi Chen', May-Su You² Hsiu-Jung Lo',3
}

1Taiwan Mycology Reference Center, National Institute of Infectious Diseases and Vaccinology, National Health Research Institutes, Miaoli, ${ }^{2}$ Institute of Molecular and Genomic Medicine, National Health Research Institutes, Miaoli, Taiwan, ${ }^{3}$ School of Dentistry, China Medical University, Taichung

The prevalence of fungal infections increases significantly in the past two decades. Major infections ( 70\%) are caused by Candida species. Candida albicans is polymorphic yeast that grows as yeast form which can extend to hyphae or pseudohyphae form. Disseminated candidiasis is associated with $30-40 \%$ mortality in severely immunocompromised patients. We have established a simple, noninvasive zebrafish egg bath infection model, defined its optimal conditions, and evaluated the model with various $C$. albicans mutant strains. Wild-type zebrafish (Danio rerio), aged approximately 8 -15 months were maintained in the zebrafish core facility at National Health Research Institutes (NHRI). One day post-fertilization, the embryos were sterilized using $0.028 \%$ chlorine bleach containing $\mathbf{0 . 0 0 1 7} \%$ sodium hypochlorite to reduce the possibility of contamination. In general, after co-incubation, non-adhered Candida cells were removed from embryos by washing with egg water 3 times. Embryos incubated in $1 \mathrm{~mL}$ of egg water in 24-well plates at $30{ }^{\circ} \mathrm{C}$ were imaged daily under an inverted microscope, with a beating heart used to indicate viability. The deletion of SAP6 did not have significant effect on the virulence. By contrast, the deletion of BCR1, CPH1, EFG1, or TEC1 significantly reduced the virulence under current conditions. Furthermore, all embryos survived when co-incubated with bcr1/bcr1, cph1/cph1 efg1/efg1, efg1/efg1, or tec1/tec1 mutant cells. The results indicated that our novel zebrafish model is timesaving and cost effective. Recently, we extended our novel zebrafish egg infection model to study the pathogenesis of other common Candida species, including C. glabrata and C. parapsilosis.

Table 1. Optimal conditions for the zebrafish egg bath infection model

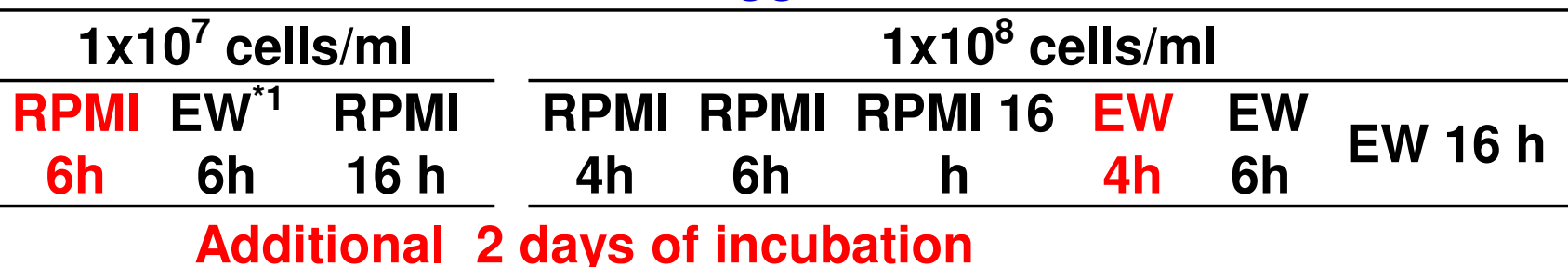

\begin{tabular}{|c|c|c|c|}
\hline Control & 100 & & \\
\hline$\overline{\text { SC5314 }}$ & 0 & & \\
\hline cph1 efg1 & 100 & & \\
\hline C. krusei & 100 & & 80 \\
\hline C. parapsilosis & 10 & & 0 \\
\hline C. glabrata & 98 & 82 & 100 \\
\hline C. tropicalis & 94 & & 56 \\
\hline
\end{tabular}

\begin{tabular}{cccccc}
100 & 100 & & 100 & 100 & \\
\hline 0 & 0 & & 10 & 33 & \\
\hline 100 & 100 & & 100 & 100 & \\
\hline 80 & 100 & 100 & 100 & 100 \\
\hline 100 & 100 & 86 & 100 & 92 \\
\hline 80 & 83 & 100 & 0 & 27 \\
\hline 82 & 100 & 100 & 100 & 100
\end{tabular}

${ }^{*} \mathrm{EW}=$ Egg water $0.03 \%$ sea salt ${ }^{* 2}$ Percentage of embryos survival rate

${ }^{*}$ Number of embryos

\section{This model can detect different level of pathogenesis within the same species.}

control

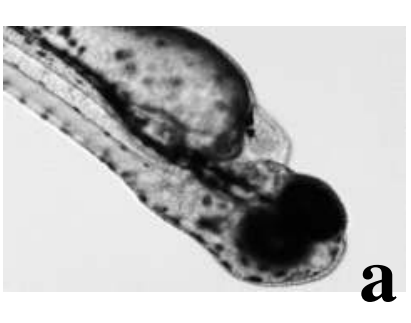

$200 \mu \mathrm{m}$

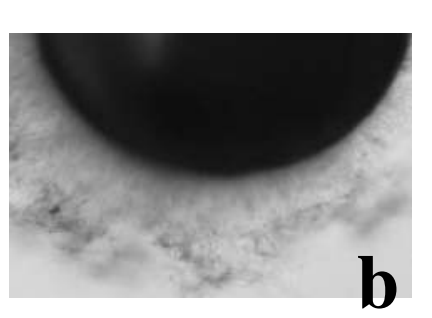

C. albicans
C. krusei
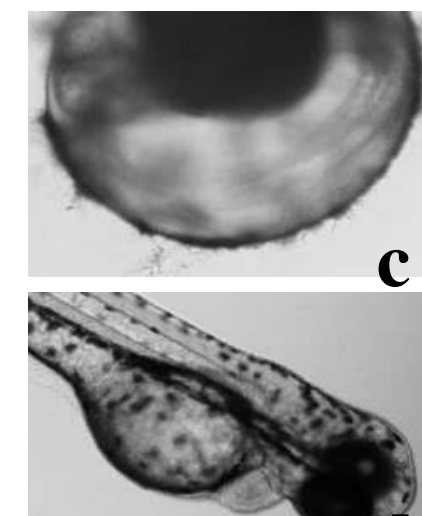

\section{c}

C. parapsilosis

C. glabrata
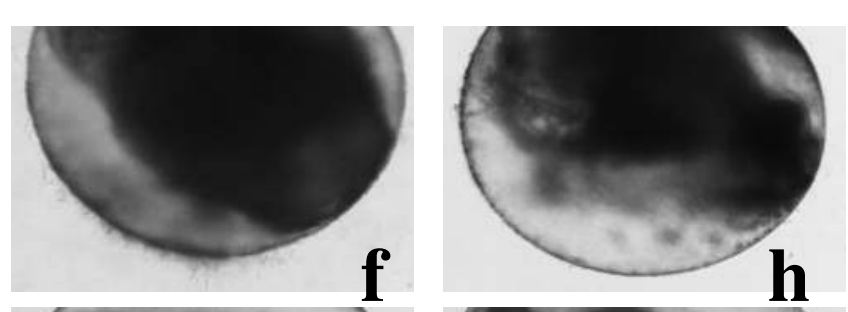

C. tropicalis

Figire. Zebrafish egg infected by pathogenic yeasts from different sources. Representative embryos were co-incubated without yeast (a) or with $1 \times 10^{7}$ cells $/ \mathrm{mL} C$. albicans (b), C. krusei (c-e), $C$. parapsilosis $(\mathrm{f}, \mathrm{g})$, and $C$. tropicalis $(\mathrm{k}-\mathrm{n})$, and in RPMI medium or in egg water (C. glabrata , $\mathrm{h}-\mathrm{j})$ at $30^{\circ} \mathrm{C}$ for $6 \mathrm{~h}$. Embryos were photographed after 2 additional days of incubation.
Table 2. Embryos survival rates after co-incubated with pathogenic yeasts

\begin{tabular}{|c|c|c|}
\hline & \multicolumn{2}{|c|}{ Additional incubation (day) } \\
\hline & 1 & 2 \\
\hline Control (a) & 100 & 100 \\
\hline SC5314 (b) & 0 & 0 \\
\hline C. krusei ATCC-Sputum (c) & 100 & 86 \\
\hline C. krusei-oral (d) & 100 & 100 \\
\hline C. krusei -oral (e) & 100 & 100 \\
\hline C. parapsilosis ATCC (f) & 20 & 0 \\
\hline C. parapsilosis -urine (g) & 100 & 90 \\
\hline C. glabrata - EW ATCC-blood (h) & 5 & 5 \\
\hline C. glabrata -oral - EW (i) & 95 & 68 \\
\hline C. glabrata -urine- EW (j) & 71 & 35 \\
\hline C. tropicalis ATCC (k) & 94 & 94 \\
\hline C. tropicalis -oral (I) & 10 & 0 \\
\hline C. tropicalis -oral (m) & 0 & 0 \\
\hline C. tropicalis -urine (n) & 89 & 89 \\
\hline
\end{tabular}

\section{CONCLUSIONS}

1. Recently, we have successfully established the zebrafish egg bath infection model in C. albicans. In the present study, we showed that this model can be extended to investigate the pathogenesis of other common pathogenic yeasts.

2. The optimal conditions are as follow: Yeasts $1 \times 10^{7}$ cells $/ \mathrm{ml}$ co-incubated with 1 day old embryos in RPMI (all tested species but $C$. glabrata) or egg water (C. glabrata) for $6 \mathrm{~h}$ at $30{ }^{\circ} \mathrm{C}$. After washed, embryos were incubated in egg water containing 0.5\% YPD for 2 days.

3. So far, we found that some $C$. parapsilosis, C. glabrata, and C. tropicalis isolates can adhere to chorion and cause embryo death.

4. We are investigating the mechanisms causing death of embryos. 